\title{
Design and Implementation Analysis of University Online Resource System
}

\author{
1,a Zhang Ke ${ }^{2, b}$ Yang Meng \\ ${ }^{1}$ Industrial and Commercial College,Hebei University, Baoding, Hebei, 071000, China \\ ${ }^{1}$ North China Electric Power University Science \& Technology College, Baoding, Hebei, 071000, \\ China \\ a,byangmeng8303@hotmail.com
}

Keywords: Online, resource system, education resource library, management information system.

\begin{abstract}
This educational resource management system can offer a resource sharing platform for students and teachers. Managing various education and teaching resources (digital resources), it realizes direct interaction between teachers and students and direct teaching management and then enhance teaching efficiency and improve work efficiency. After system performance test with testing tool, we get that the system is capable to maintain the average response time of 0.05 second with concurrent operation of 100 users, and the system interface functions well, which can satisfy the basic needs of users.
\end{abstract}

\section{Introduction}

As an emerging educational pattern, online teaching has become one of the most important educational patterns except for recruitment undergraduate education [1, 2]. Till now, 6 years have passed since generation and universities works on online teaching have developed to 67 ones with 9 categories and 153 majors from the original 4 schools. The swift development speed means the general acceptance of online school and this new education pattern offers a convenient approach for self-training and permanent education. At present, the total number of national-wide online teaching students nearly doubled comparing with last year, increasing to nearly 2.5 million from 1.6 million [3]. With the development of online teaching, network resource construction has become the key point. Online teaching offers an efficient convenient approach of educating, learning, management and service by internet, breaking restrictions of time and space and promoting education development.

However, online teaching in our country is still at relative low level. From the claw we may infer the lion. Online teaching development is not long but fast in our country. Till now, various educational institutions and academies have web portals and develop several teaching assistance system. The educational resource library begins to take shape but without effective information management tools $[5,6]$. Generally, online teaching construction lacks of standards and normalizations and techniques hasn't formed a complete system, resulting in incapability of constructing high-quality online teaching educational resources system $[7,8]$. There are numerous ICP offering various education community platforms or market services, but actual courses contents are still deficient and can’t complete online teaching service.

Thus, this paper aims at developing a resource system platform suitable for students at different levels. To ensure the best use of the system, we research search algorithm and ASP caching technology and put forwards solutions to searching problems and resource utilization problems. All users can perform instructional interaction on the system platform.

\section{Frame Analysis of Educational Resource Management System}

According to the analysis process bottom up, middle school online teaching resource library system is consistent by the following parts in general. Its framework includes campus network common resource foundation database, personal resource libraries of teachers and students, resource 
administration platform, user personalization operation module, resource application and access interface ( which can be subdivide into teacher module and student module) etc. Its structure diagram is shown in Figure1.

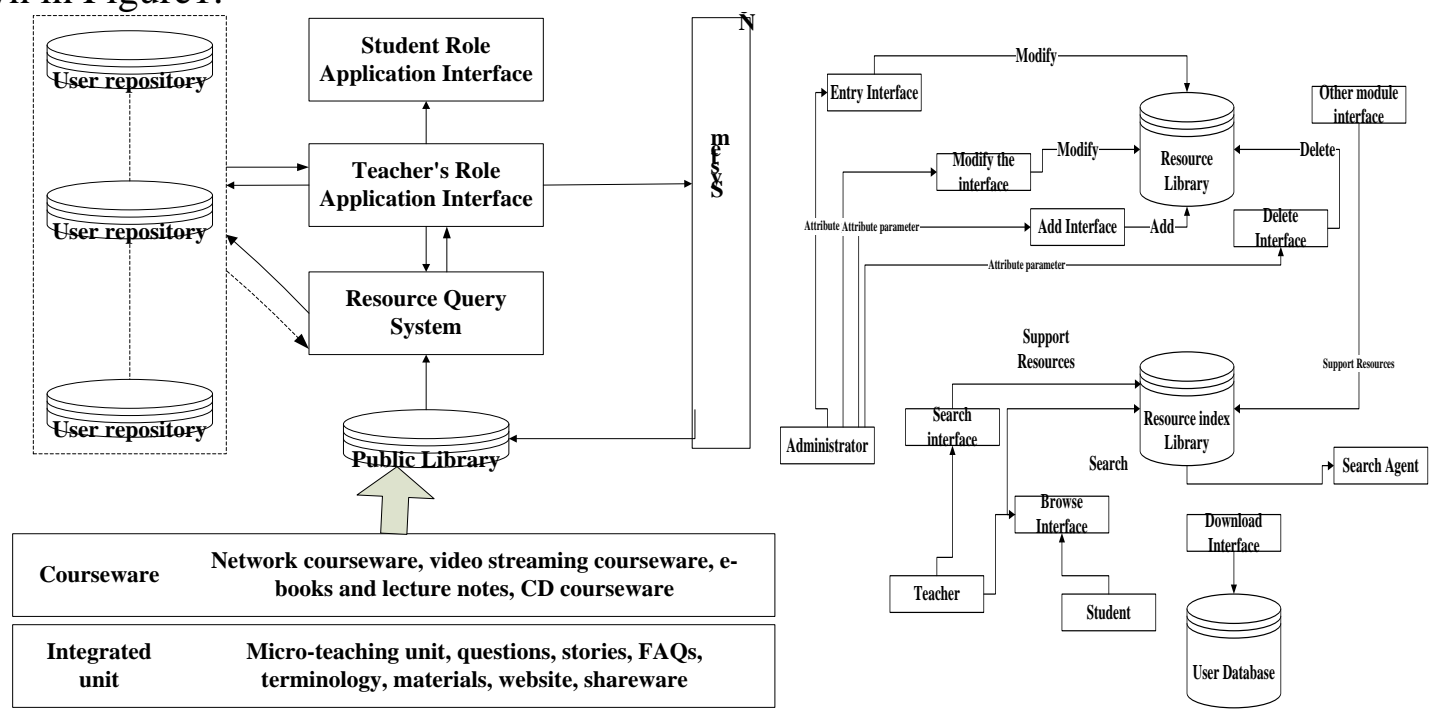

Figure 1 General Framework of Educational Resource Management System Figure 2: Resource Administration Transaction Processing model

Among them, resources in common resource library are under united control of the system and any user has no right to manage and operate this part of resources. Differences in data content and visit particle size divide the database into basic resources, integrated cell and teaching courseware. Basic resource contains elementary units constituted by educational resources, such as text resources, multimedia resources, URL bases, common problem bases, terminology Database, and basic symbols and graphic library of subjects. We store combined resources constituted by elementary units in integrated cells, like item bank, Microteaching library, case library, reference material database and sharing software library etc. Courseware resources include all kinds of electronic teaching plans, electronic lectures, instruction videos, e-books, disk courseware etc. In order to offer personalized online teaching as well as make it convenient to share educational resources according to course requirements, the system contains personal resource library, namely every teachers can manage their own resources in file format as well as download resources in common resource library as personal resources. Administer authorized personal resources can turn into common resources.

\section{Overall Resource Framework Analysis}

Processing Procedure of Educational Resource Management Practice:The construction of educational resource library and its management system is a long-term task, which also determines the processing procedure of resource management transaction differs with common information management system. We must conduct integrated analysis from the initial phase of library construction and determine the processing logic of user function modules in the educational resource management system by process of library construction.

Users of resource management system are divided into registered users (system administers, students, teachers) and non-registered users (visitors). Differences in rights of authority determine logic differences after users' login the system and they are operated in different resource spaces. According to the above process of resource construction, we can summarize several main logic process of the system: Store educational resources. Administers can login homepage and hide administration menu or check webpages and resource use condition as a visitor. Administers can complete resource storage related work through type-in interface or manage stored resources by related operation interface (modification interface, delete interface, addition interface) so as to accomplish resource audit and update as well as get statistical overview of resources. As the main users, teachers and students visit the resource library by corresponding interfaces, for example, 
teachers can search by resource search interface after setting up association attributes (the resource library sets up numerous resource indexes to accelerate searching speed to solve the efficiency problem of extensive search). The main procedures of users visiting and operating resource management transaction are shown in Figure 2.

Framework Analysis of Functional Structure:According to the analysis of processing model, the main functions of educational resource management system are normal search, advanced search, discipline classification, resource classification, grade classification, user resource library, the third party resource library and other functions. As for the system users, the primary function of educational resource management system is to offer resource search and dynamic management function. The main functions of middle-school educational resource management system this paper developed are shown in Figure 3.
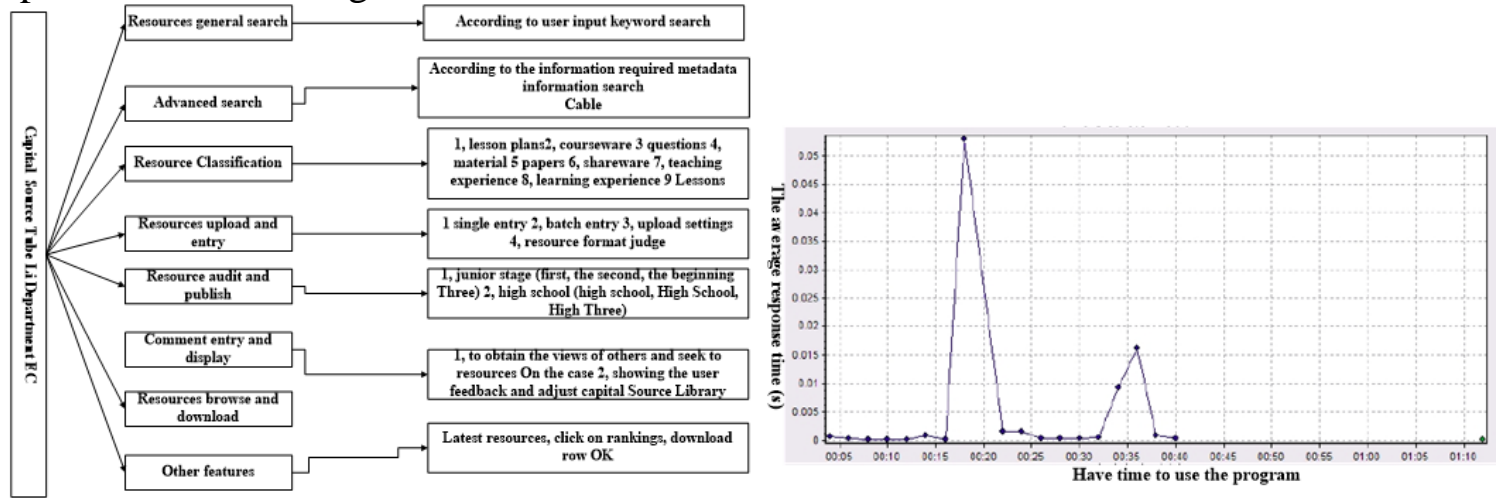

Figure 3 Structure of Resource Management System Figure 4: Average Response Time

Resource search distinguishes by limits of authority after user login. The system offers corresponding resource searching interface and module in line with user characteristics and sets up different searching procedures, which sharing similar search functions. Users can also create and manage their resources when uploading resources beforehand. The system has to support the batch input to diminish the work of library initialization. Besides, to ensure the uploaded resource qualities and prevent from malicious upload, the system needs to audit the submitted educational resources, thus all operations have to be completed in resource list. The system needs to provide with well browse interface and download support module to make it convenient for users acquiring educational resources. It positions resources and displays their corresponding characteristics by system navigation bars and searching modules. To help users make pointed selection and use resources, the system allows legal users (mainly teachers) to make comments on the resource. System administers can summarize the grade of one resource by comprehensive comment situations. This contributes to routine adjustment and resource update. And as for resources with low comments or few visit and use, we can eliminate or adjust.

\section{Algorithm Design and Code implementation}

If administer input wrong information and doesn't realize before adding but want to modify it after adding successfully, he should click the modifying and deleting function key of this resource. Data are deleted or acquired by findById and findAll in DriverDAO. After entering course resource management interface, information of all available resources can be revealed by findAll; and when click delete, appointed curriculum resources and disk ID can be acquired by findById and this is operated by sql statements.

Users have to input related disk information when searching. The system will inquire information as requested. The main design codes are shown as follows:

protected void btnSearch_Click(object sender, EventArgs e)

\{

string str $=$

ConfigurationManager.ConnectionStrings["guangpansystemConnectionString1"].ToS

tring(); 
SqlConnection con = new SqlConnection(str);

con.Open();

string book = this.txtBookname.Text.Trim().ToString();

SqlDataAdapter sda = new SqlDataAdapter("select * from guangpan where

name like '\%" + guangpan + "\%"', con);

DataSet set $=$ new DataSet();

sda.Fill(set);

this.GridView1.DataSource = set;

\}

this.GridView1.DataBind();

Normal users have no right to modify any resources and information and their only rights are browsing and searching. The system will prompt when one operates beyond his rights.

Key codes are shown as follows.

if(session.getAttribute("user_id")==null||session.getAttribute("user_pwd")==nul

1||session.getAttribute("user_status")==null||session.getAttribute("user_nickname")==

null $)\{<$ div align="center" $><$ span class="text11" $>$ you are normal user $</$ span $><$ br $>$

$<$ br $><$ ahref="\#"class="link5"onClick="javascript:window.open('../login.jsp', ",

'width=404, height=235')" $>\operatorname{login}</ \mathrm{a}></ \operatorname{div}>$ \}

This system conducts user concurrent testing on resource download. User logins the system, uploads a file, adds news, searches a resource, check an announcement and then log out the system. The total number of user login is 100 with 10at first and adding 5 users every 2 seconds. The test result is shown in Figure 4 and Figure 5.

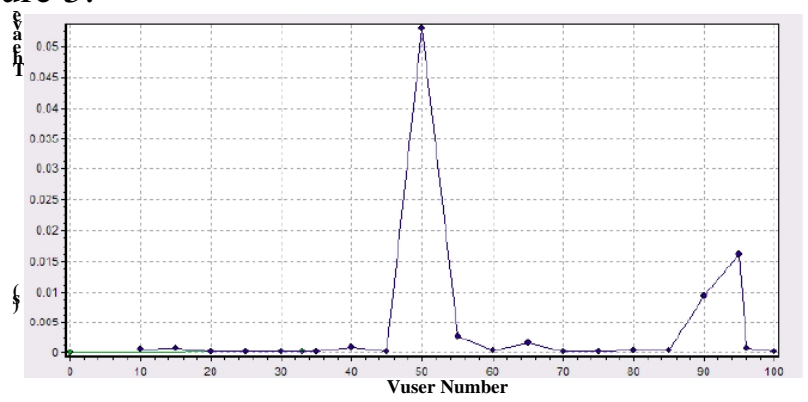

Figure 5 Response Time in Different loads

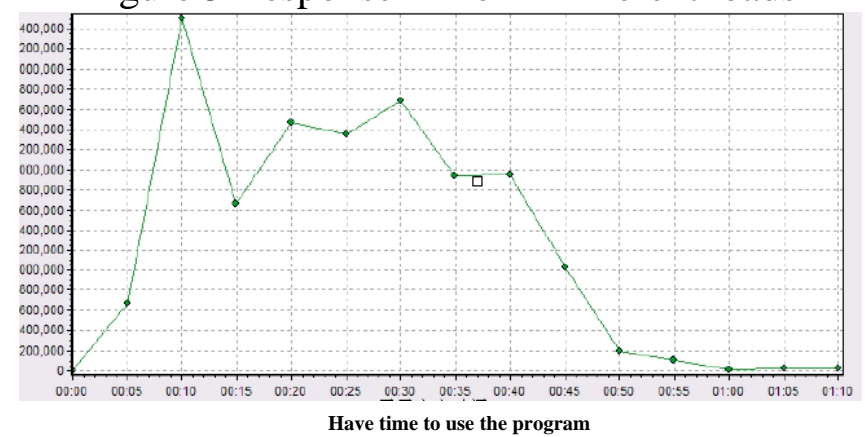

Figure 6 Handling Capacity

In the light of the above Portfolio performance test principle, this paper simulates maximum user number in a certain period to execute the concurrent execution situation of the main modules respectively. The highlight test is to get the handling capacity and response time of the system under high-capacity operation. The system function in maximum portfolio is got by integrated analysis of execution index and resources monitoring. The test result is shown in Figure 6.

The involved business processes in the educational resource management system design are according to the process in real life so that users can use the system more naturally and conveniently. The functional test proves the final design effect of this system is identical to the expected effect, which means the development of system function accords with users' needs. After system performance test with testing tool, we get that the system is capable to maintain the average response 
time of 0.05 second with concurrent operation of 100 users, and the system interface functions well, which can satisfy the basic needs of users.

\section{Conclusions}

With the popularization of educational informationization technology in various middle and primary schools, a lot of schools have developed teaching assistance software like teaching affair administration, examination management, high-quality curriculum website etc., which managing various teaching information and resource stored in campus network. Educational resource construction is the foundation of online teaching. The construction of educational resources network platform provides wide middle and primary schools, teachers and students with comprehensive application service to realize educational resource share. This educational resource management system can offer students and teachers a resource sharing platform to manage various education and teaching resources (digital resources) so as to realize direct interaction between teachers and students and direct teaching management and then enhance teaching efficiency and improve work efficiency.

\section{Reference}

[1] Dong Liang, Lu Xianghua, Huang Lihua. Source Model Analysis of ASP Platform Operation [J] Journal of Management, 2007, (01): 39-42.

[2] ]Yang Gaixue, Interpreting informationized Educational Resources [J1. E-education Research,2009(03),12- 14.

[3] Cheng Jiejing. The Theory Research on Digital Building of Educational Information Resources in Network Environment[J] Modern Educational Technology,2009(03),94-97..

[4] Meng Xiangzeng Content-based FLASH online Education Resource Searching Study[J] E-education Research,2009(09),77-80

[5] Sheng Quan, Zhang Yunzhu, Cao Chunming. New Development of Middle-School Education Resource Management Construction[J]. China E-educational Technology, 2011, 17(5):43-45.

[6] Si Yulv. Study on E-Learning Resource Library Construction [J]. Journal of Shenyang Normal University, 2011, 22(5): 39-41

[7] Yang Zongkai. Application of Information Expression Technology Based on XML in Education Resource Register System[J]. Computer Engineering, 2012. 33(10): 190-192

[8] Tao Qiuxia. Design and Application of Web-based Educational Resource Management System [J].Shanxi Science and Technology, 2012, 26(5):51-52 